\title{
Avaliação dos parâmetros físico químicos e microbiológicos do leite cru informal envasados em garrafas - PET comercializados em bairros periféricos em um município da região norte do Brasil
}

Evaluation of physical, chemical and microbiological parameters of informal raw milk packaged in bottles - PET marketed in peripheral neighborhoods in a municipality in the northern region of Brazil Evaluación de parámetros físicos, químicos y microbiológicos de leche cruda informal embotellada - PET comercializada en barrios periféricos de un municipio del norte de Brasil

Sérgio Luis Melo Viroli ORCID: https://orcid.org/0000-0003-3982-1183 Instituto Federal do Tocantins, Brasil E-mail:viroli@ifto.edu.br Fernando Morais Rodrigues ORCID: https://orcid.org/0000-0002-3750-5593 Instituto Federal do Tocantins, Brasil E-mail: fernandomorais@ifto.edu.br

Sérgio Guimarães Viroli ORCID: https://orcid.org/0000-0002-6558-5391 Universidade Federal do Tocantins, Brasil E-mail: sergio.viroli@mail.uft.edu.br

Nelson Pereira Carvalho ORCID: https://orcid.org/0000-0002-7637-1292 Instituto Federal do Tocantins, Brasil

E-mail: nelson.pereira.carvalh@gmail.com

Henrique Avelino Duarte ORCID: https://orcid.org/0000-0003-2331-5171 Instituto Federal do Tocantins, Brasil

E-mail: henrique.duarte@estudante.Ifto.edu.br

Tiago Teixeira Alves

ORCID: https://orcid.org/0000-0002-7807-2085

Universidade Federal do Tocantins, Brasil E-mail: tiagotavessof@gmail.com João Vitor Vivan

ORCID: https://orcid.org/0000-0001-6826-6049 Instituto Federal do Tocantins, Brasil E-mail: jvitorvivan.jvv@gmail.com

Matheus Lisboa Ramos

ORCID: https://orcid.org/0000-0002-1648-6733 Instituto Federal do Tocantins, Brasil E-mail: matheus.lisboas13@gmail.com

Karoline Pereira Feitosa

ORCID: https://orcid.org/0000-0002-2925-814X Instituto Federal do Tocantins, Brasil

E-mail: karolyne18ximenes@gmail.com

\section{Resumo}

O leite é um dos alimentos mais completos da natureza em razão de seus constituintes nutricionais, tornando-se suscetível à contaminação por microrganismos patogênicos. O estudo objetivou avaliar os parâmetros físico-químicos acidez, densidade, lipídios, proteínas, pH e microbiológicos através da contagem padrão em placas do leite cru informal envasado em garrafas-PET comercializado e consumido em quatro bairros distintos de um município localizado na região norte do Brasil. As amostras foram armazenadas em caixa térmicas contendo gelo e transportadas para os Laboratórios de Microbiologia e Análises de Alimentos do Instituto Federal do Tocantins - IFTO campus Paraíso do Tocantins, para realização das análises físico-químicas e microbiológicas. As amostras foram codificadas em: A, B, C e D. O delineamento experimental foi inteiramente casualizado (DIC) com quatro (4) tratamentos e dez (10) repetições. A fim de verificar ocorrências de diferença entre as médias das variáveis respostas aplicou-se a analise de variância ANOVA e o teste de Tukey ao nível de 5\% de significância. Análise de Componentes Principais (ACP), 
Técnica de análise multivariada, foi utilizada e analisou os dados nos quais os tratamentos estão relacionados por variáveis de resposta inter-relacionadas, agrupando as variáveis físico-químicas similares. A avaliação físico-química da acidez do leite apresentou valores médios $(0,19 \mathrm{~g}$ ácido láctico/100 mL) em desconformidade com os valores

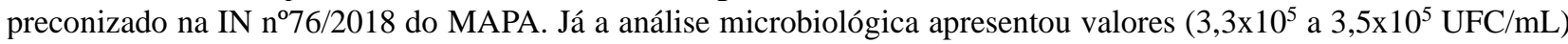
acima dos estabelecidos pela legislação, indicando que o leite foi obtido e comercializado em condições higiênicosanitárias impróprias. Sugere-se maior atenção dos órgãos de fiscalização, a fim de impedir a compra pelos consumidores e possível contaminação alimentar, além de, campanhas de esclarecimentos sobre os riscos e possíveis problemas de saúde advindos do consumo de leite informal não pasteurizado.

Palavras-chave: Qualidade do leite; Análise de componentes principais; Segurança alimentar; Pasteurização.

\begin{abstract}
Milk is one of nature's most complete foods due to its nutritional constituents, making it susceptible to contamination by pathogenic microorganisms. The study aimed to evaluate the physical-chemical parameters acidity, density, lipids, proteins, $\mathrm{pH}$ and microbiological through the standard counting in plates of informal raw milk bottled in PET bottles sold and consumed in four different neighborhoods of a municipality located in the northern region of Brazil. The samples were stored in a thermal box containing ice and transported to the Microbiology and Food Analysis Laboratories of the Instituto Federal do Tocantins - IFTO, Paraíso do Tocantins campus, for physical-chemical and microbiological analyses. The samples were coded in: A, B, C and D. The experimental design was completely randomized (DIC) with four (4) treatments and ten (10) replications. In order to verify occurrences of differences between the means of the response variables, ANOVA analysis of variance and Tukey's test were applied at a 5\% significance level. Principal Component Analysis (PCA), a multivariate analysis technique, was used and analyzed the data in which treatments are related by interrelated response variables, grouping similar physicochemical variables. The physical-chemical evaluation of milk acidity showed average values $(0.19 \mathrm{~g}$ lactic acid/100 $\mathrm{mL})$ in disagreement with the values recommended in MAPA IN $n^{\circ} 76 / 2018$. The microbiological analysis showed values (3.3x105 to $3.5 \times 105 \mathrm{CFU} / \mathrm{mL}$ ) above those established by legislation, indicating that the milk was obtained and marketed under improper hygienic-sanitary conditions. It is suggested greater attention from inspection bodies, in order to prevent the purchase by consumers and possible food contamination, in addition to campaigns to clarify the risks and possible health problems arising from the consumption of informal unpasteurized milk.
\end{abstract}

Keywords: Milk quality; Principal component analysis; Food safety; Pasteurization.

\title{
Resumen
}

La leche es uno de los alimentos más completos de la naturaleza debido a sus componentes nutricionales, haciéndola susceptible a la contaminación por microorganismos patógenos. El estudio tuvo como objetivo evaluar los parámetros físico-químicos acidez, densidad, lípidos, proteínas, $\mathrm{pH}$ y microbiológicos a través del conteo estándar en platos de leche cruda informal embotellada en botellas PET vendidas y consumidas en cuatro barrios diferentes de un municipio ubicado en la región norte. de Brasil Las muestras fueron almacenadas en caja térmica con hielo y transportadas a los Laboratorios de Microbiología y Análisis de Alimentos del Instituto Federal do Tocantins - IFTO, campus Paraíso do Tocantins, para análisis físico-químicos y microbiológicos. Las muestras se codificaron en: A, B, C y D. El diseño experimental fue completamente al azar (DIC) con cuatro (4) tratamientos y diez (10) repeticiones. Para verificar la ocurrencia de diferencias entre las medias de las variables de respuesta, se aplicó el análisis de varianza ANOVA y la prueba de Tukey al 5\% de significación. Se utilizó el Análisis de Componentes Principales (PCA), una técnica de análisis multivariado, y se analizaron los datos en los que los tratamientos están relacionados por variables de respuesta interrelacionadas, agrupando variables fisicoquímicas similares. La evaluación físico-química de la acidez de la leche mostró valores medios $(0,19 \mathrm{~g}$ ácido láctico/100 $\mathrm{mL})$ en desacuerdo con los valores recomendados en el MAPA IN n76/2018. El análisis microbiológico mostró valores (3,3×105 a 3,5x105 UFC/mL) por encima de los establecidos por la legislación, lo que indica que la leche fue obtenida y comercializada en condiciones higiénicosanitarias inadecuadas. Se sugiere mayor atención por parte de los órganos de control, a fin de prevenir la compra por parte de los consumidores y la posible contaminación de los alimentos, además de campañas para esclarecer los riesgos y posibles problemas de salud derivados del consumo informal de leche no pasteurizada.

Palabras clave: Calidad de la leche; Análisis de componentes principales; Seguridad alimenticia; Pasteurización.

\section{Introdução}

A produção de leite brasileira ocupa a $4^{\mathrm{a}}$ posição no ranking mundial, gera 6,7 milhões de empregos diretos e indiretos e realiza uma considerável função socioeconômico no Brasil (Santos et al., 2021). O êxodo rural é minimizado através de atividade leiteira, ocasionada pela ampliação da produção resultante do crescimento do rebanho e particularmente aumento da produtividade (Ulisses et al., 2022). 
Embora a produção de leite seja significativa para o agronegócio brasileiro, o leite in natura cru mantido sob refrigeração pode apresentar impasses no que se refere aos padrões de qualidade (Ulisses et al., 2022). Segundo Silvestrin et al. (2022) e Ulisses et al. (2022), o leite cru é capaz de veicular microrganismos deterioradores e patogênicos atraídos durantes os estágios da cadeia produtiva, sendo necessário a realização de teste para certificar a sua qualidade. Devido ao enorme consumo in natura e abundante variedade de produtos lácteos no mercado oferecidas ao consumidor, a origem e a qualidade do leite cru são primordiais tanto do ponto de vista econômico quanto de saúde pública (Araújo et al., 2021).

Segundo Espindola et al. (2020), mediante a alta relevância da qualidade do leite, foi criada a Instrução Normativa IN n 76 de 26 de novembro de 2018, que regulamenta os parâmetros higiênico-sanitários e classificação em diferentes tipos, conforme a qualidade, manipulação, armazenamento e comercialização do leite. Ainda segundo os autores, essa instrução normativa impacta positivamente no mercado nacional e internacional, sendo que a legislação exige um leite com alta qualidade higiênica-sanitária possibilitando sua exportação.

Segundo Fagnani e Zanon, (2019), a possibilidade de infecção correlacionado ao consumo de leite cru são evidentes e inquestionáveis. Ainda segundo os autores, o leite cru comercializado direto do produtor para o consumidor, sem pasteurização, oriundo de bovinos clinicamente sadios podem estar susceptíveis a contaminação por microrganismos causadores de doenças, dentre elas a síndrome hemolítico -urêmica. Segundo Pereira et al. (2019), entre os microrganismos associados as enfermidades transmitidas pelo leite cru, a bactéria Staphylococcus aureus é uma das mais importantes pois é altamente patogênica ao homem. Logo, o consumo de leite cru, sem tratamento térmico, pode ser um perigo para a saúde pública. Segundo Santos et al. (2021) e Ströher et al. (2021) o leite cru in natura para consumo humano deve ter sua sanidade atestada através de um rígido controle de qualidade dos parâmetros físico-químicos e microbiológicos atendendo aos padrões exigidos pela legislação pertinente, isento de resíduos de qualquer natureza, adulterantes, conservantes e valores de vestígios de antibióticos estabelecidos em lei.

Fagnani e Zanon, (2019), afirmam que embora haja perigos à saúde humana ocasionado pela ingestão de leite não pasteurizado, muitos indivíduos consomem o leite cru através da distribuição ilegal no mercado informal contrariando as restrições governamentais. Ressalta-se que a praticidade, os preços acessíveis, as questões culturais e o desconhecimento dos perigos que esse tipo de produto pode representar a saúde do consumidor, são fatores contribuintes para a continuidade ou aumento da comercialização desse leite chamado de "clandestino" ou "informal.

O comércio clandestino de leite cru in natura acondicionados em garrafas plásticas descartáveis PET, transportado em motos sem refrigeração e controle higiênico sanitário ocorre habitualmente em cidades brasileiras (Silva et al., 2017). Segundo Motta et al., (2015), 20 a 30\% do leite produzido no Brasil é comercializado informalmente, sem inspeção sanitária ou tratamento térmico adequado, em recipientes de vidro ou plásticos reutilizados de outros produtos. Já para Filho et al., (2016), a ingestão de leite não pasteurizado em determinadas cidades brasileiras é um habito relacionado a causas culturais, regionais e sociais explicado pela funcionalidade, baixo poder aquisitivo do produto e principalmente a conviçcão de que o leite adquirido diretamente do produtor é mais "saudável" que o pasteurizado. De acordo com Sovinski et al., (2014) a manutenção habitual da comercialização direta do leite cru informal na zona urbana pelos produtores rurais ou leiteiros que realizam venda direta ao consumidor, em pontos comerciais varejistas de alimentos, que desconhecem os riscos potenciais que podem ser veiculados e mantém este ciclo de comercialização informal.

Segundo Fagnani e Zanon, (2019), a pasteurização obrigatória e os regulamentos de venda de leite cru estão relacionados a diminuição de surtos de enfermidade ocasionada pela ingestão leite. Antes da década de 1950, cerca de $25 \%$ de todas as infecções transmitidas por alimentos eram relacionadas ao leite. Ainda segundo os autores, após a inserção dos regulamentos que recomendam a pasteurização do leite, reduziu para $1 \%$ o envolvimento do leite com os surtos de doenças transmitidas. No entanto, nos últimos anos esse número parece estar aumentando à medida que é permitida a venda legal de 
leite cru. A manutenção da informalidade do comércio do leite cru in natura gera consequências econômicas, sociais e prejuízos à saúde da população.

Diante do exposto, o presente estudo teve como objetivo avaliar os parâmetros físico-químicos e microbiológicos do leite cru informal comercializado e consumido em quatro (4) bairros periféricos no município de Paraíso do Tocantins, Estado do Tocantins, indicando as condições higiênico-sanitárias e aptidão para o consumo.

\section{Metodologia}

Essa pesquisa baseada em estudo laboratorial e experimental com utilização método quantitativo para obtenção de dados. O método quantitativo utiliza a estatística para verificação da hipótese (Pereira, \& Shitsuka, 2018)

O estudo foi realizado nos laboratórios de Microbiologia e Analise de Alimentos do Curso Superior em Tecnologia de Alimentos do Instituto Federal de Educação, Ciência e Tecnologia do Tocantins (IFTO) do Campus Paraíso, localizado no Km 480, Distrito Agroindustrial na Cidade de Paraíso do Tocantins, Tocantins, Brasil.

\subsection{Localização e amostragem do leite cru analisado.}

Durante os meses de fevereiro a novembro de 2019 no intervalo das 7h às 9h da manhã, em dias diferentes da semana foram coletadas amostras mensais de leite cru informal em diferentes pontos de comercialização localizados em quatro (4) bairros periféricos na cidade de Paraíso do Tocantins totalizando 40 amostras pesquisadas. A aquisição das amostras foi realizada por meio de compra do leite (Figura 1).

Figura 1. Localização dos pontos de coleta do leite informal na cidade de Paraíso do Tocantins - TO.

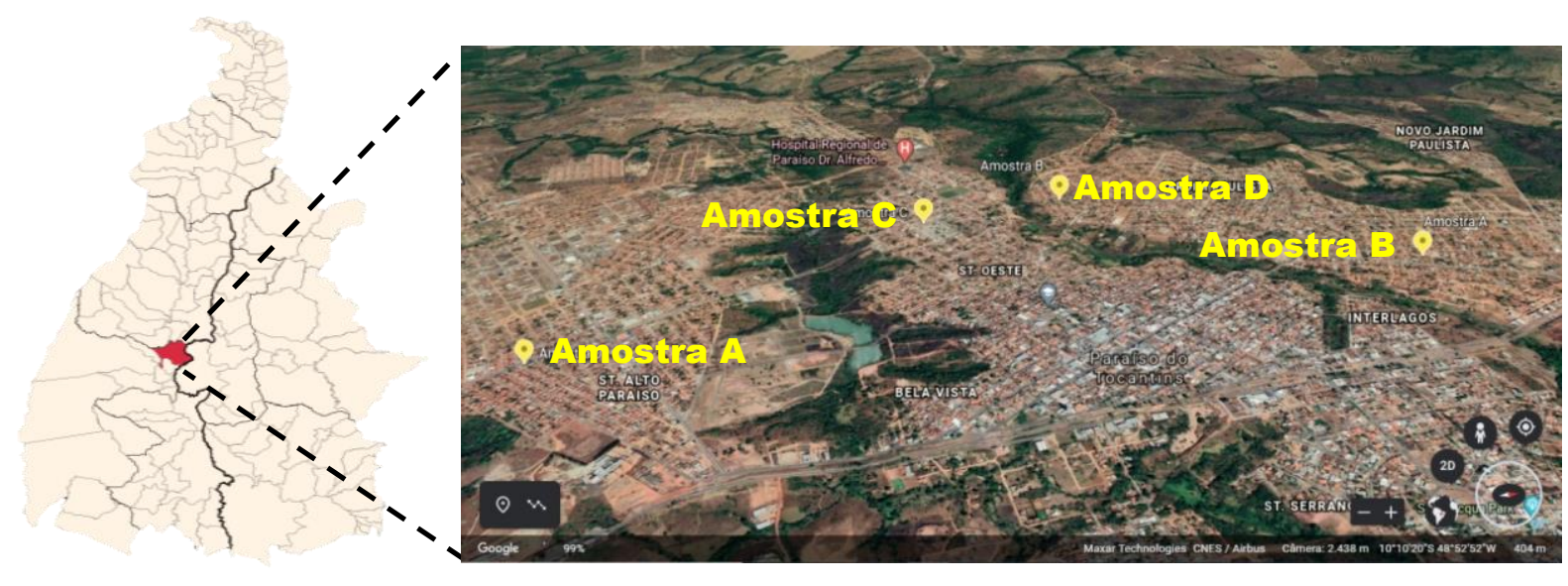

Fonte: Autores (2022).

O leite foi coletado diretamente dos pontos de vendas, bairros A, B, C e D, em suas embalagens de comercialização, garrafas PET, (Figura 2) e analisadas dentro de um período de máximo, 24h após as coletas. 
Figura 2. Pontos de venda de comercialização do leite cru informal na cidade de Paraíso do Tocantins - TO.

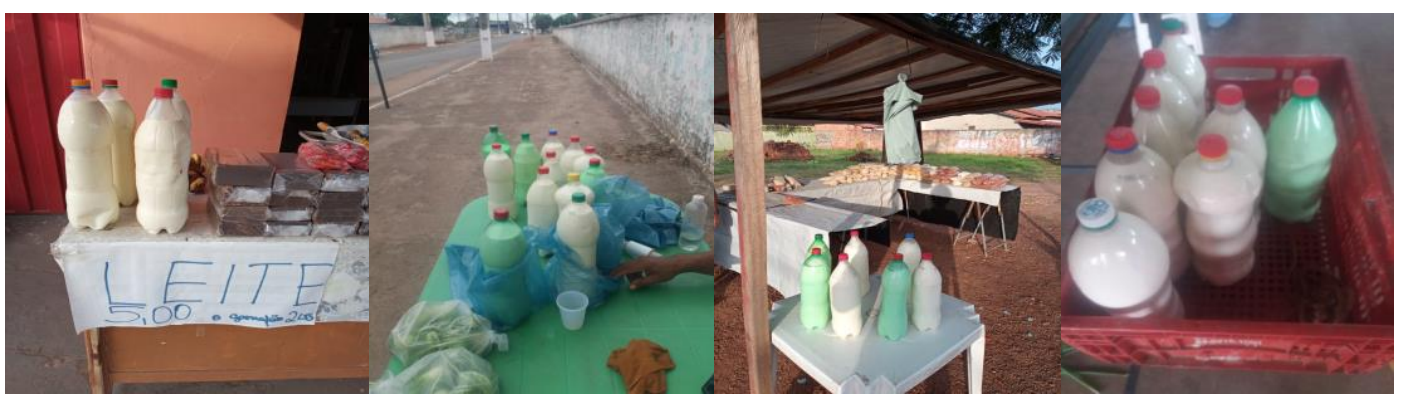

Fonte: Autores (2022).

As amostras foram armazenadas em caixa térmicas contendo gelo e transportadas para Instituto Federal do Tocantins - IFTO campus Paraíso do Tocantins, para realização das análises físico-químicas e microbiológicas. As amostras foram codificadas em: A, B, C e D.

\subsection{Caracterização do leite cru informal comercializado.}

\subsubsection{Analises Físico químicas}

As análises físico-químicas de acidez, densidade, lipídeos, proteína e potencial hidrogenionico-pH foram realizadas em triplicada no Laboratório de Análise de Alimentos do IFTO/Campus Paraíso e seguiram os procedimentos metodológicos abaixo:

- Acidez: determinada por titulação, sendo expressa em g/100g de ácido láctico (IAL, 2008);

- Densidade: apontada através de leitura direta em termolactodensímetro de Quevenne, com escala entre 1,015 a 1,040 g.cm-3 (IAL, 2008);

- Lipídios: quantificados pela extração direta em Soxhlet (IAL, 2008);

- Proteínas: realizadas pela técnica de Kjeldahl e o fator de 6,25 para conversão em proteína pela determinação do nitrogênio total (IAL, 2008);

- Potencial hidrogeniônico (pH): por leitura direta com utilização de potenciômetro digital (IAL, 2008).

\subsubsection{Analises microbiológicas}

As analises microbiológicas para contagem padrão de placas (CPP) foi realizada de acordo com Manual de Métodos Oficiais para Análise de Alimentos de Origem Animal (Brasil, 2019), com resultados expresso em UFC/mL e os resultados comparados com a Instrução Normativa No 76 de 26 de novembro de 2018 (Brasil, 2018).

\subsubsection{Analise estatística}

O delineamento experimental foi inteiramente casualizado (DIC) com quatro (4) tratamentos e dez (10) repetições, totalizando 40 amostras pesquisadas. A fim de verificar se houve diferença significativa entre os resultados aplicou-se a Analise de variância (ANOVA) e entre as médias das variáveis de resposta o teste de Tukey ao nível de 5\% de significância. Técnica de análise multivariada foi utilizada para melhorar a análise estatística dos resultados, como a Análise de Componentes Principais (ACP), que analisou os dados nos quais os tratamentos estão relacionados por variáveis de resposta inter-relacionadas, onde o objetivo foi agrupar as variáveis físico-químicas em função da similaridade. A ACP foi realizada nos dados padronizados para evitar o efeito dos diferentes níveis de grandeza das variáveis de resposta. A padronização dos dados 
de cada variável de resposta foi feita mediate a subtração de cada valor pela sua média dividida pelo erro padrão. Todas as análises estatísticas foram realizadas com o programa SISVAR versão 5.6 (Ferreira, 2019).

\section{Resultados e Discussão}

\subsection{Analises físico químicas}

Na Tabela 1 é possível observar os resultados obtidos para analises físico química de acidez, potencial hidrogeniônico $(\mathrm{pH})$, densidade, lipídeos e proteínas. As amostras de leite cru informal comercializadas em quatro (4) bairros periféricos da cidade de Paraíso do Tocantins, apresentaram valores de acidez fora dos padrões preconizados pela legislação. Não houve diferença significativa $(\mathrm{p}>0,05)$ para os testes físico químicos de $\mathrm{pH}$, densidade, lipídeos e proteína

Tabela 1. Médias das análises físico-químicas.

\begin{tabular}{|c|c|c|c|c|c|}
\hline \multirow[b]{2}{*}{ Amostra* } & \multirow[b]{2}{*}{$\begin{array}{l}\text { Acidez } \\
\text { (g/100g) }\end{array}$} & \multicolumn{4}{|c|}{ Parâmetros Físico químicos } \\
\hline & & pH & $\begin{array}{l}\text { Densidade } \\
(\mathrm{g} / \mathrm{mL})\end{array}$ & $\begin{array}{l}\text { Lipídeo } \\
\text { (g/100g) }\end{array}$ & $\begin{array}{r}\text { Proteína } \\
(\mathrm{g} / 100 \mathrm{~g})\end{array}$ \\
\hline $\begin{array}{l}\text { A } \\
\text { B } \\
\text { C } \\
\text { D }\end{array}$ & $\begin{array}{c}0,1905^{\mathrm{a}} \pm 0,01 \\
0,1911^{\mathrm{b}} \pm 0,01 \\
0,1904^{\mathrm{b}} \pm 0,01 \\
0,1901^{\mathrm{ab}} \pm 0,01\end{array}$ & $\begin{array}{l}6,62^{\mathrm{a}} \pm 0,02 \\
6,64^{\mathrm{a}} \pm 0,02 \\
6,63^{\mathrm{a}} \pm 0,02 \\
6,61^{\mathrm{a}} \pm 0,02\end{array}$ & $\begin{array}{l}1,029^{\mathrm{a}} \pm 0,01 \\
1,029^{\mathrm{a}} \pm 0,01 \\
1,030^{\mathrm{a}} \pm 0,01 \\
1,028^{\mathrm{a}} \pm 0,01\end{array}$ & $\begin{array}{l}3,41^{\mathrm{a}} \pm 0,01 \\
3,44^{\mathrm{a}} \pm 0,01 \\
3,40^{\mathrm{a}} \pm 0,01 \\
3,32^{\mathrm{a}} \pm 0,01\end{array}$ & $\begin{array}{l}3,03^{\mathrm{a}} \pm 0,02 \\
2,94^{\mathrm{a}} \pm 0,01 \\
3,10^{\mathrm{a}} \pm 0,01 \\
2,91^{\mathrm{a}} \pm 0,01\end{array}$ \\
\hline $\begin{array}{c}\text { Instrução Normativa } \mathrm{N}^{0} \\
76 / 2018\end{array}$ & $\begin{array}{c}0,14 \text { a } 0,18 \\
\text { (g ácido láctico/100mL) }\end{array}$ & ------------ & $\begin{array}{c}1,028 \text { a } 1,034 \\
(\mathrm{~g} / \mathrm{mL})\end{array}$ & $\begin{array}{c}\text { mínimo } \\
\text { 3,0(g/100g) }\end{array}$ & $\begin{array}{c}\text { mínimo } \\
2,9(\mathrm{~g} / \mathbf{1 0 0 g})\end{array}$ \\
\hline
\end{tabular}

Legendas: pH: potencial hidrogeniônico; *Médias seguidas da mesma letra na vertical não apresentam diferença significativa (p > 0,05) pelo teste de Tukey. Fonte: Autores (2022).

$\mathrm{Na}$ Tabela 1, estão representadas as médias das análises físico-químicas do leite informal comercializado e consumidos em quatro (4) bairros periféricos A, B, C e D da cidade de Paraíso do Tocantins

Todas as amostras apresentaram valores médios de acidez $(0,19 \mathrm{~g} / 100 \mathrm{~g})$ acima do estabelecido pela legislação vigente (Brasil, 2018). Esses resultados indicam que provavelmente houve falha na etapa de refrigeração logo após a ordenha, falta de higiene durante a obtenção da matéria prima e/ou envase em recipientes mal higienizado. Ressalta-se que a ausência de refrigeração favorece a predominância de microrganismos mesófilos que atuam intensamente na fermentação da lactose, produzindo ácido láctico e acidificação do leite. Estudo realizado sobre análise do leite produzido na cidade de Santana do Livramento - Rio Grande do Sul encontraram 40\% das amostras com acidez fora do padrão estabelecido pela legislação (Arbello et al., 2021). Silva et al. (2017), realizando avaliação físico-química de leite in natura comercializado informalmente no sertão paraibano verificou que 37,5\% das amostras apresentaram valores para a acidez em discordância com a legislação vigente. Estudos conduzidos por Oliveira et al. (2020), avaliando a qualidade do leite cru e prevalência de mastite no município de Mossoró-RN encontraram valores para o teste de acidez dentro do limite permitido pela legislação, sendo as duas primeiras coletas no limite máximo exigido. Araújo et al. (2021) realizando ensaios para determinação de fraudes em leite cru, obtiveram resultados para a análise de acidez dentro do preconizado pela legislação. A acidez do leite é utilizada como indicativo da qualidade, quando relacionada ao estado de conservação, em função da relação entre a disponibilidade de lactose e a produção de ácido láctico, devido à ação de microrganismos deteriorantes que convertem a lactose do leite em ácido láctico. Refrigeração inadequada após a ordenha ou utensílios e equipamentos mal higienizados, comercialização em garrafas PET, pontos de vendas em calçadas de residências e comércios e exposição prolongada ao sol são fatores que contribuem para o aumento da acidez do leite.

A perda da estabilidade térmica pode ocorrer em leites não ácidos. Segundo Silvestrin et al., (2022) esta situação evidencia a incidência de Leite Instável Não Ácido (LINA), ocorrendo no momento em que o leite apresenta instabilidade da caseína, porém sua acidez está dentro do padrão estipulado pela legislação. Sendo assim o leite LINA pode ser entendido como 
leite ácido, punir o fornecedor de leite e desestabilizar-se durante ao tratamento térmico industrial. Ainda segundo Silvestrin et al., (2022), destaca que o excesso de sais minerais na composição do leite diminui a quantidade de água disponível para as proteínas se ligarem, facilitando a precipitação da caseína frente à prova do álcool, características genéticas do animal, região, estágio de lactação, época do ano, adversidades fisiológicas, nutricionais e metabólicas são possíveis causas da ocorrência do leite LINA.

A densidade variou de 1,028 a 1,029 g/mL. A Legislação brasileira, Instrução Normativa 76/2018 (Brasil, 2018), admite uma variação para a densidade entre 1,028 a $1,034 \mathrm{~g} / \mathrm{mL}$ de leite a $15^{\circ} \mathrm{C}$ (BRASIL, 2018). A avaliação da densidade é utilizada na detecção da adulteração do leite, pois valores menores do que o limite mínimos indicam adição de água, e valores maiores que o limite máximo indica a inclusão outras substâncias ou desnate do leite. Segundo Melo, (2018), a densidade é uma propriedade física que expressa a associação entre massa e volume e está relacionada diretamente com o peso específico do leite, ao sofrer adulteração apresenta um peso específico inferior de 1,028g/mL. Silvestrin et al., (2022) constatou valores para as densidades de acordo com os padrões estabelecidos pela legislação em análise da qualidade físico-química do leite cru entregue a uma cooperativa beneficiadora do município de Juína-MT. Marques et al (2019) avaliando a densidade do leite cru refrigerado de produtores assistidos pela EMATER/RS no município de São Luiz Gonzaga (RS) encontrou resultados para todas as amostras dentro do preconizado pela Instrução Normativa $n^{\circ} 76$ (BRASIL, 2018). Em estudos conduzidos por Souza et al. (2018) analisando a densidade em leite in natura comercializado informalmente no município de Imperatriz MA, obteve $30 \%$ das amostras analisadas com valores abaixo da média preconizada exigida pela legislação (Brasil, 2018). Ulisses et al., (2022), avaliando a densidade em leite cru refrigerado em propriedades rurais pertencentes ao município de Alegre/ES obteve com resultado $11,12 \%$ das amostras em discordância com o valor preconizado na legislação. Os resultados obtidos por Souza et al. (2018) e Ulisses et al (2022) desconcordam com os valores encontrados para a densidade no presente estudo. Ainda segundo Ulisses et al., (2022), amostras com densidades fora dos limites estabelecidos pela a Instrução Normativa 76/2018 (Brasil, 2018), gera rejeição do leite cru refrigerado no instante da recepção, pois o leite cru esta densidade alterada devido a inclusão de água ou desnate nas propriedades pelo produtor.

O teor de lipídeos encontrado nesse estudo variou de 3,3 a 3,4 g/100g estando de acordo com o recomendado pela legislação vigente. Os lipídios (gordura) atribuem consistência e rendimento ao leite e podem ser influídos pela separação da nata do leite, idade, raça ou período de lactação, nutrição, alimentação, estresse térmico, depressão ou falta de conforto do animal. Teores de lipídios dentro dos padrões estabelecidos pela Instrução Normativa $n^{\circ}$ 76/2018. do Ministério de Agricultura e Pecuária e Abastecimento, superiores aos limites mínimos de 3,0 g/100g, foram encontrados por Guimaraes et al, (2020) em estudos sobre a qualidade do leite in natura refrigerado em quatro propriedades rurais do Município de Rio Verde GO. Em análises referentes ao teor de lipídeos, Ulisses et al (2022) obteve todas as amostras analisadas apresentando valores acima do mínimo de 3,0\%, estando, portanto, em concordância com a legislação. Guimaraes et al, (2020) e Ulisses et al (2022) obtiveram resultados similares aos deste estudo. Os resultados encontrados por Souza et al (2018), para teor de lipídeos foram observados que $20 \%$ das amostras analisadas encontravam se fora da faixa preconizada pela legislação. Marques et al. (2020) realizando estudos sobre o teor de matéria gorda em leite cru refrigerado de produtores no município de São Luiz Gonzaga (RS), encontrou $20 \%$ das amostras analisadas em desconformidade aos requisitos impostos pela legislação. Ainda segundo Marques et al. (2020), a gordura é o componente que mais varia e é de extrema importância para obtenção de derivados do leite. Os resultados encontrados por Souza et al. (2018) e Marques et al (2020) para a determinação de lipídios divergem dos resultados encontrados nesse estudo. Segundo Ulisses et al (2022), o teor de lipídeos determina o valor do leite em indústrias de derivados do leite, pois a gordura é utilizada para produzir creme de leite e manteiga. Devido à alta valorização do lipídio no leite, é fundamental a definição exata da quantidade de gordura para a indústria leiteira e produtores de leite.

Silvestrin et al. (2022) e Espindola et al. (2020) afirmam que percentual de fibras na dieta bovina é um fator que 
influencia diretamente na fração desse integrante no leite, pois maior teor de fibras na alimentação do animal, maior será a percentual de gordura do leite podendo variar entre 2 a 3 unidades percentuais.

No que diz respeito ao conteúdo proteico nesse estudo, os valores variaram de 2,9 g/100g a 3,0 g/100g estando em conformidade com o estipulado na Instrução Normativa 76/2018 (Brasil, 2018). Os valores para a determinação de proteínas encontrados no presente estudo são maiores do que os encontrados por Assis et al (2021) que variaram de 2,18 g/100g a 2,94 $\mathrm{g} / 100 \mathrm{~g}$ em leite bovino in natura provenientes do Ceará e menores do que encontrado por Arbello et al (2021), que variaram de $3,12 \mathrm{~g} / 100 \mathrm{~g}$ a 3,48 g/100g, analisando leite produzido na cidade de Santana do Livramento - Rio Grande do Sul. Souza et al (2018) realizando analise de proteína em leite in natura comercializado informalmente no município de Imperatriz, obteve $40 \%$ das amostras com valores muito abaixo do limite mínimo preconizado na legislação brasileira ( 2,9 g de proteínas/100 g). Espindola et al. (2020), analisando a qualidade do leite cru refrigerado produzido na microrregião de Pires do Rio, Goiás, obteve 3,18\% de proteínas em leites obtidos em ordenha manual e 3,25\% de proteínas em leites através d ordenha mecânica. Ambos os valores encontrados por Espindola et al estão de acordo com os valores proteicos estipulado na legislação brasileira.

Segundo Silvestrin et al., (2022), a alteração do teor de proteínas no leite pode estar associada ao estado sanitário, a raça dos animais, estágio de clima, tipo de alimentação fornecida, lactação, entre outros fatores. Ainda segundo os autores, a fração de proteínas no leite é um critério utilizados por alguns lacticínios como medida de qualidade do leite e razão para pagamento diferenciado aos produtores de leite.

Em relação ao potencial hidrogeniônico $(\mathrm{pH})$ os valores variaram de 6,62 a 6,63. A legislação brasileira através da Instrução Normativa n 76 de 26 de novembro de 2018 do Ministério da Agricultura Pecuária e Abastecimento (MAPA) não estabelece padrão para valor do potencial hidrogeniônico $(\mathrm{pH})$ que deve apresentar o leite cru refrigerado. Portanto, neste estudo foi aplicado como parâmetro o valor estipulado pela literatura. Segundo Ströher et al. (2021), relata que alguns autores consideram o potencial hidrogeniônico $(\mathrm{pH})$ para o leite cru refrigerado variando entre 6,60 a 6,75 ou 6,60 a 6,80 . Souza et al (2018) avaliando leite informalmente no município de Imperatriz, obteve 15\% dos resultados para os testes de pH abaixo do valor de 6,5. Marques et al (2019), avaliando leite cru no município de São Luiz Gonzaga RS, também obteve um resultado menor 6,51 para a análise de $\mathrm{pH}$. Ainda segundo Marques et al., o leite com pH entre 6,5 e 6,6 é ligeiramente ácido podendo ser leite em inicio da lactação, com colostro, ou princípio de desenvolvimento fermentativo. Segundo Ströher et al. (2021), o aumento da temperatura tente a diminuir o valor do $\mathrm{pH}$ devido a dissociação da água, separação do difosfato de tri cálcio e formação de ácido.

\subsection{Analises Microbiológicas}

Com relação aos resultados obtidos na análise da contagem padrão em placas - CPP, verificaram-se valores variando de 3,3 x $10^{5} \mathrm{UFC} / \mathrm{mL}$ a 3,5 x $10^{5} \mathrm{UFC} / \mathrm{mL}$. Segundo a Instrução Normativa no 76 (BRASIL, 2018), o limite máximo é de 3,0 x $10^{5} \mathrm{UFC} / \mathrm{mL}$. Na Tabela 2 encontram-se os resultados das análises microbiológicas obtidos nessa pesquisa e os valores de referências preconizado na Instrução Normativa $N^{\circ} 76$ de 26 de novembro de 2018. As amostras analisadas não diferiram entre si $(\mathrm{p}>0,05)$. 
Tabela 2. Valores médios da analises de contagem padrão em placas.

\begin{tabular}{|c|c|}
\hline Amostra & $\begin{array}{c}\text { Contagem Padrão em Placas } \\
\text { (UFC/mL) }\end{array}$ \\
\hline $\mathrm{A}$ & $3,3 \times 10^{5 \mathrm{~A}}$ \\
\hline $\mathrm{B}$ & $3,5 \times 10^{5 \mathrm{~A}}$ \\
\hline $\mathrm{C}$ & $3,5 \times 10^{5 \mathrm{~A}}$ \\
\hline $\mathrm{D}$ & $3,4 \times 10^{5 \mathrm{~A}}$ \\
\hline Instrução Normativa $N^{\circ} 76 / 2018$ & $\leq 3,0 \times 10^{5} \mathrm{UFC} / \mathrm{mL}$ \\
\hline
\end{tabular}

Medias seguidas pela mesma letra nas colunas não diferem entre si. Teste de Tukey ( $\mathrm{p} \geq 0,05)$. Fonte: Autores (2022).

A Tabela 2 demostra os valores médios da analises de contagem padrão em placas de leite cru refrigerado comercializado e consumido em quatro (4) bairros periféricos no município de Paraíso do Tocantins -TO.

Marque et al (2020) encontraram resultados elevados para a contagem padrão de placas, variando de $1,78 \mathrm{x} 10^{5} \mathrm{UFC} / \mathrm{mL}$ a 4,8 × $10^{6} \mathrm{UFC} / \mathrm{mL}$ totalizando $70 \%$ das amostras analisadas fora do limite estipulado pela legislação. Trindade et al. (2018) pesquisando a qualidade microbiológica do leite cru comercializado informalmente no município de Rio Pomba, encontraram valores elevados para CPP variando de $3 \times 10^{4} \mathrm{UFC} / \mathrm{mL}$ a $8 \times 10^{8} \mathrm{UFC} / \mathrm{mL}$. Ströher et al. (2021), relata que ao analisar amostras de leite de propriedades leiteiras, 23,3\% dessas propriedades apresentaram inconformidade em relação aos valores estipulados na legislação brasileira para a contagem padrão de placas. Arbello et al (2021) realizando analise microbiológica em leite cru produzido na cidade de Santana do Livramento RS observaram que as amostras oriundas de propriedades rurais apresentaram 26,66\% dos resultados com alta CPP.

Segundo Marques et al (2020), o desconhecimento ou a falta de aplicação dos conceitos a respeito das boas práticas de ordenha pelos produtores de leite acarreta a obtenção de leite de baixa qualidade microbiológica com valores abaixo do que preconiza a legislação. Ainda segundo os autores, gestos simples como higienização do úbere do animal antes da ordenha, equipamentos e utensílios limpos e higienizados, boas condições de uso dos equipamentos, higienização da sala de ordenha e das mãos, uso de roupas limpas e refrigeração do leite minimizam a contaminação dos microrganismos e melhora a qualidade do leite obtido. Segundo Paludetti et al. (2017) o resfriamento do leite e o armazenamento refrigerado após a ordenha das vacas são fundamentais para redução do percentual ou proliferação bacteriana no leite. De acordo com Arbello et al (2021), a não conformidade com os requisitos de higiene representam risco para a saúde dos consumidores, pois o leite cru pode veicular microrganismos patogênico causadores de enfermidades transmitidas durante o consumo pelos seres humanos. Os autores ainda argumentam que a situação do contágio das enfermidades em humanos é mais frequentes em indivíduos que consomem o leite cru em vez do consumir leite pasteurizado. Baixas ou mínimas contagem de microrganismo no leite cru indicam a ocorrência de boas práticas no decorrer da ordenha e boa higienização durante o processo de obtenção do leite.

\subsection{Analise de componentes Principais (ACP)}

A Análise de Componentes Principais (ACP) (Figura 3 e 4) forneceu uma interpretação simplificada das relações entre as variáveis de resposta bem como das amostras, facilitando a correlação entre elas e permitindo identificar quais tratamentos são caracterizados por determinadas variáveis. 
Figura 3. Análise de componentes principais: representação das variáveis físico-químicas.

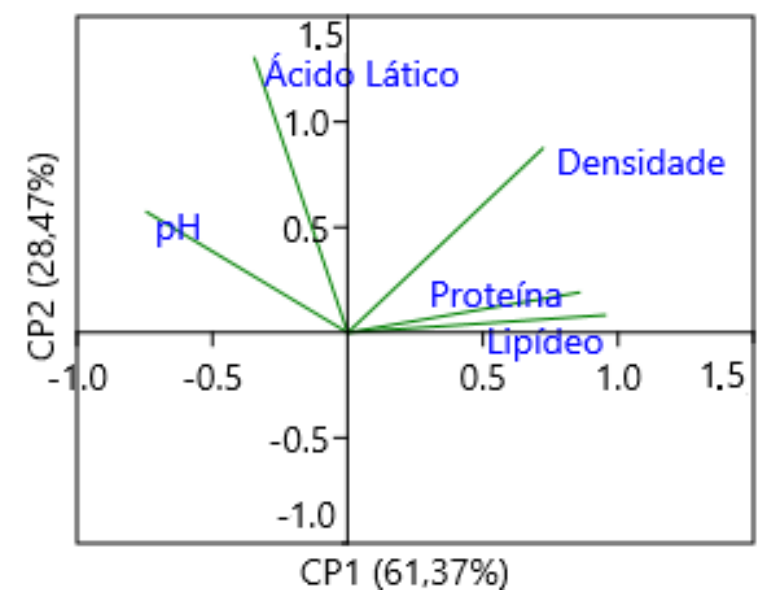

Fonte: Autores (2022).

A Figura 3 apresenta as componentes principais (CP1) e (CP2), exibindo os seus respectivos pesos, possibilitando uma visualização dos principais agrupamentos no conjunto de variáveis.

Figura 4. Análise de componentes principais das amostras: representação das amostras.

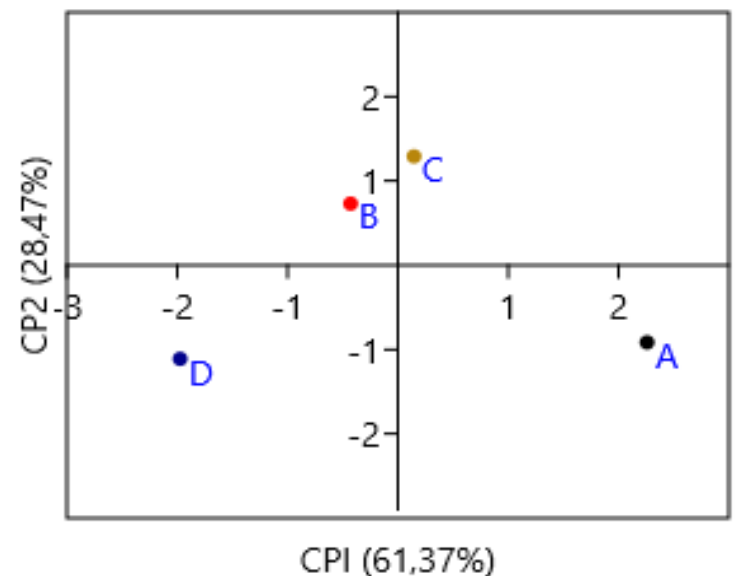

Fonte: Autores (2022).

A Figura 4 demostra a visualização das amostras de leite cru informal comercializado nos Bairros A, B, C e D nos bairros periféricos na Cidade de paraíso do Tocantins.

As Figuras 3 e 4 demostram os valores dos scores do primeiro componente principal (PC1) que explica 61,37\% e pelo segundo componente (PC2) 28,47\%. Neste experimento, a soma dos dois primeiros componentes (CP1 e CP2) explicaram $89,84 \%$ da variabilidade dos dados.

De acordo com a Figura 4, a amostra C está relacionado com os maiores teores de densidade e proteína, por estar situado em quadrante equivalente a tais atributos, conforme as Figuras 4 e 3. Já a amostra A relacionou-se com os maiores níveis de lipídeo. Adicionalmente, nota-se que a amostra B está relacionada com os maiores teores de acidez, por estarem situados em quadrantes próximo a tal atributo. Ainda de acordo com a figura a amostra D relacionou-se com os menores valores para todos as variáveis avaliadas. 


\section{Conclusão}

A avaliação da qualidade do leite cru informal comercializado nos quatro (4) bairros no município de Paraíso do Tocantins, TO, através de análises físico-químicas e microbiológicas, apresentou irregularidades para acidez e contagem de microrganismos frente à IN 76/2018, evidenciando o perigo que a ingestão deste produto expõe a saúde do consumidor. Constatou-se também a necessidade de refrigeração e cuidados com a obtenção e transporte do leite cru, que resulta em acidificação do produto e contagens de microrganismos acima do permitido pela legislação vigente. Sendo assim, a comercialização do leite informal é inadequada, pois os resultados das análises microbiológica evidencia a precariedade do produto.

Sugere-se maior atenção dos órgãos de fiscalização, a fim de impedir a compra pelos consumidores e possível contaminação alimentar e uma campanha de esclarecimento dos possíveis problemas para a saúde do consumidor ao ingerir leite não pasteurizado.

\section{Referências}

Araújo, J. de, Gräff, C. A., \& Silvestre, W. P. (2021). Qualitative tests for the determination of fraud in raw milk: evaluation of the influence of analytical parameters of the tests and the stability of the samples as a function of time and preservation form. Research, Society and Development, 10(11), e450101119860. https://doi.org/10.33448/rsd-v10i11.19860

Arbello, D. D. R., Braccini, V. P., Escalona Jiménez, M., Erhardt, M. M., \& Richards, N. S. P. dos S. (2021). Análise microbiológica e físico-química do leite produzido na cidade de Santana do Livramento. Research, Society and Development, 10(6), e24310615561. https://doi.org/10.33448/rsd-v10i6.15561

Assis, R. C. de, Nogueira, M. D. de A., Pinheiro, A. D. V., Galdino, R. S., Almondes, K. G. de S., \& Maia, C. S. C. (2021). Determinação de selênio em leite bovino in natura provenientes do Ceará - Brasil. Research, Society and Development, 10(6), e48010616011. https://doi.org/10.33448/rsd-v10i6.16011

Brasil. (2018). Ministério da Agricultura Pecuária e Abastecimento. Instrução normativa n. 76, de 26 de novembro de 2018 . Aprova Regulamentos Técnicos que fixam a identidade e as características de qualidade que devem apresentar o leite cru refrigerado, o leite pasteurizado e o leite pasteurizado tipo A, na forma desta Instrução Normativa e do Anexo Único. Diário Oficial da União, 30/11/2018 | Edição: 230 | Seção: 1 | Página: 9.

Brasil. Ministério da Agricultura, Pecuária e Abastecimento. (2019). Manual de métodos oficiais para análise de alimentos de origem animal. Ministério da Agricultura, Pecuária e Abastecimento. Secretaria de Defesa Agropecuária. - 2. ed. Brasília.

Candido, F. dos S., Mendonça, T. O., Zanini, D. da S., Meneguelli, M., Berndt, F. M., \& Muniz, I. M. (2020). Qualidade microbiológica do leite cru refrigerado. Research, Society and Development, 9(11), e839119584. https://doi.org/10.33448/rsd-v9i11.9584

Condé, P. R., Pinto, C. L. de O., Gandra, S. O. da Silva., Silva, R. R. da, \& Martins, M. L. (2018). Temperatura de armazenamento e qualidade microbiológica do leite cru granelizado na região de Rio Pomba, Minas Gerais. Revista do Instituto de Laticínios Cândido Tostes, 73(3), 149-161. DOI:https://doi.org/10.14295/2238-6416.v73i3.699

Couto, K. S., \& Chinelate, G. C. B. (2017). Avaliação de leite cru comercializado informalmente na cidade de Garanhuns - PE - Brasil. Revista Brasileira De Agrotecnologia, 7(1), 09-12. https://www.gvaa.com.br/revista/index.php/REBAGRO/article/view/4708

Espindola, W. R., Nascente, E. de P., Urzêda, M., Teodoro, J. V. da S., Gonçalves, G. B., Castro, R. D. de, Martins, M. E. P., \& Souza, W. J. de. (2020). Qualidade do leite cru refrigerado produzido na microrregião de Pires do Rio, Goiás, Brasil. Research, Society and Development, 9(7), e153973958. https://doi.org/10.33448/rsd-v9i7.3958

Fagnani, R., Eleodoro, J. I., \& Zanon, E. O . (2019). Milk-borne infections awareness and the health status of consumers: An on-line survey International Dairy Journal, 96, p.85-92. https://doi.org/10.1016/j.idairyj.2019.04.013

Ferreira, D. F. (2019). Sisvar: A computer analysis system to fixed effects split plot type designs. Revista Brasileira de Biometria, 37(4), 529-535. DOI: https://doi.org/10.28951/rbb.v37i4.450

Filho, W. L. G. S., Dias, C. S., Melo, J. D. G., Santos, E. C. C., Silva, A. S., \& Araújo, A. P. Avaliação físico-química do leite cru em Redenção no Pará. Revista Tecnologia \& Ciências Agropecuárias, 10(5), 29-34, jul. 2016.

Guimarães, G. M., Mateus, L. S., Moraes, A. I. P., Costa, W. S. da, Soares, N. R., Santos, L. S. dos, \& Santos, P. A. dos. (2020). Qualidade do leite in natura perante a instrução normativa IN 76 do Ministério da Agricultura, Pecuária e Abastecimento do Brasil. Research, Society and Development, 9(9), e262996746. https://doi.org/10.33448/rsd-v9i9.6746

Instituto Adolfo Lutz-IAL. (2018). Métodos físico-químicos para análise de alimentos. São Paulo-SP: IAL.

Jiménez, M. E., Braccini, V. P., Seibt, A. C., Machado, L. V., Erhardt, M. M., Silva, G. P. da., \& Richards, N. S. P. dos S. (2021). Características socioeconômicas da produção e parâmetros de qualidade do leite cru refrigerada no Município de Santa Maria, RS, Brasil. Research, Society and Development, 10(6), e13510615562. https://doi.org/10.33448/rsd-v10i6.15562 
Marques, T., Pires, P., \& Leães, F. (2020). Avaliação da qualidade de leite cru refrigerado de produtores assistidos pela EMATER/RS no município de São Luiz Gonzaga (RS). Revista Principia - Divulgação Científica E Tecnológica Do IFPB, 1(52), 117-128. http://dx.doi.org/10.18265/15170306a2020v1n52p117-128

Melo, C. W. B. de., Barbosa, F. R., \& Pereira, D. E. (2018). Avaliação da qualidade do leite cru refrigerado obtido em propriedades rurais localizadas no estado da Paraíba. Revista Brasileira de Produtos Agroindustriais, 20(2),.137-142. 137

Monte, A. M., Sousa, A. W. B. de, Ramos, L. de S. N., Cunha, L. S., Lima, L. R. do N., Diniz, H. da S., Negreiros, I. F. L., \& Moreira Filho, M. A. (2021). Contagem de células somáticas em leite cru refrigerado de produtores individuais através de métodos rápidos. Research, Society and Development, 10(6), e2210615160. https://doi.org/10.33448/rsd-v10i6.15160

Motta, R. G., Silva, A. V., Giuffrida, R., Siqueira, A. K., Paes, A. C., Motta, I. G., Listoni, F. J.P., \& Ribeiro, M. G... (2015). Indicadores de qualidade e composição de leite informal comercializado na região Sudeste do Estado de São Paulo. Pesq. Vet. Bras., 35(5), p. 417-423. 10.1590/S0100736X2015000500005

Oliveira, P. V. C., Neto, E. S. L., Lucena, N. M., Abrantes, M. L., Silva, J. B. A \& Neto, C. O. A. (2020). Avaliação da qualidade do leite cru e prevalência de mastite no município de Mossoró-RN. Brazilian Journal of Development, 6(8), 64027-64042. https://doi.org/10.34117/bjdv6n8-728

Paludetti, L. F., Kelly, A. L., O’brienb., Jordan, K. , \& Gleeson, D. (2018). The effect of different precooling rates and cold storage on milk microbiological quality and composition. Journal of Dairy Science 101(3). p. 1921-1929 https://doi.org/10.3168/jds.2017-13668

Pereira, A. S., Shitsuka, D. M., Pereira, F. J., \& Shitsuka, R. (2018). Metodologia da pesquisa científica. UFSM

Pereira, M. F. B. C., Gomes, P. W. P.1, Simões, M. C., Martins, L. H. da S., \& Sarmento, P. S. de M. (2019). Avaliação microbiológica no leite de vaca in natura e pasteurizado comercializado na cidade de Tucuruí, Pará. Biota Amazônia, 9(3), 52-56. http://dx.doi.org/10.18561/21795746/biotaamazonia.v9n3p52-56.

Santos, C. G. dos., Dallago, G. M., Dumont, M. A., Figueiredo, L. V. de, Duarte, M. R., Boari, C. A., Castro, G. H. de F., \& Santos, R. A. dos. (2021). Composition and microbiological quality of raw milk refrigerated in community tanks. Research, Society and Development, 10(11), e208101119574. https://doi.org/10.33448/rsd-v10i11.19574

Silva, G. W. N., Oliveira, M. P., Leite, K. D., Oliveira, M. S., \& Sousa, B. A. de A. (2017). Avaliação físico-química de leite in natura comercializado informalmente no sertão paraibano. Revista Principia. 1(35), p. 34-41. http://dx.doi.org/10.18265/1517-03062015v1n35p34-41.

Silvestrin, P. D., Sodré, L. W. de B., \& Oliveira, A. P. de. (2022). Análise da qualidade físico-química do leite crú entregue a uma cooperativa beneficiadora do município de Juína-MT. Research, Society and Development, 11(1), e59811125476. https://doi.org/10.33448/rsd-v11i1.25476

Souza, J. V., Paiva, B. L. F., Santos, A. F. C., Fontenele, M. A., Araújo, K. S. da S., \& Viana, D. C. (2018). Avaliação dos parâmetros físico-químicos do leite "in natura" comercializado informalmente no município de Imperatriz MA. Revista Brasileira de Agropecuária Sustentável, 8(4), 1-6. https://doi.org/10.21206/rbas.v8i4.3064

Sovinski, A. I., Cano, F. G., Raymundo, N. K. L., Barcellos, V. C., \& Bersot, L. dos S. (2014). Situação da comercialização do leite cru informal e avaliação microbiológica e físico-química no município de Cafelândia, Paraná, Brasil. Arq. Ciênc. Vet. Zool., 17(3) 161-165. https://doi.org/10.25110/arqvet.v17i3.4938

Ströher, J. A., Nunes, M. R. dos S., Santos Junior, L. C. O. dos., \& Schröetter, L. F. (2021). Análise da qualidade do leite de produtores do Norte do Estado do Rio Grande do Sul (RS). Research, Society and Development, 10(11), e415101119580. https://doi.org/10.33448/rsd-v10i11.19580

Trindade, L. C. A., Martins, M. L., Martins, J. M., \& Martins, A, D. de O. (2018). Qualidade de leite cru comercializado informalmente no município de Rio Pomba, MG. Higiene Alimentar, 32(284/285), 72-76, 2018. http://docs. bvsalud.org/biblioref/2018/11/964916/284-285-setout-2018-72-76.pdf.

Ulisses, A. de F., Píccolo, M. da P., Rangel, O. J. P., Santos Júnior, A. C., \& Maia Júnior, J. de A. (2022). Leite cru refrigerado: qualidade microbiológica, físico-química e detecção de resíduos de antibióticos. Research, Society and Development, 11(1), e48111123708. https://doi.org/10.33448/rsd-v11i1.23708 